\title{
Cyclostationary noise analysis of superregenerative oscillators
}

\author{
Silvia Hernández, Sergio Sancho, Almudena Suárez \\ Department of Communications Engineering, University of Cantabria, Spain
}

\begin{abstract}
A rigorous analysis of noise effects in super regenerative oscillators (SRO) operating in linear mode is presented. The analysis takes into account the cyclostationary nature of the SRO response to the noise sources, due to the effect of the quench signal. It is based on the determination of an envelope-domain linear-time-variant (LTV) transfer function with respect to each noise source, plus the application of a detailed stochastic analysis of the SRO output. Initially, the autocorrelation of the output signal is calculated, which varies at two different time scales, and is periodic with respect to the quench signal, so it can be expressed in terms of the frequency-dependent harmonic components of the LTV transfer functions. This enables the calculation of the output spectral density, depending on these harmonic components. Once the spectral density is known, the signal-to-noise ratio can be obtained in a straightforward manner. The analysis method has been validated with both independent circuit-level simulations and measurements.
\end{abstract}

Keywords - superregenerative oscillator, stability, noise.

\section{INTRODUCTION}

Superregenerative oscillators (SROs) use the exponential growth of an oscillation signal to obtain high gain amplification. Due to the high gain values attained, SROs have been used to replace amplifier chains in receivers [1]-[2] and, more recently, have also been implemented in active transponders [3]. The oscillation is controlled by a quench signal that periodically switches the oscillator on and off (Fig. 1), by shifting the critical pair of complex-conjugate poles from the left-hand side of the complex plane (LHS) to the right-hand side (RHS) and then back to the LHS. The SRO is sensitive to the input signal only during a fraction of the quench-signal period, located about the time value at which the critical pair of complex-conjugate poles crosses to the RHS [1]-[2]. The signal grows as long as this critical pair of poles is located on the RHS, so the maximum output amplitude is obtained when the poles cross again to the LHS [1]-[2]. Thus, the SRO responds with an output pulse, controlled by the quench signal (Fig. 1). The input signal provides an initial condition that places the system at a certain level of the initial transient and, in the so-called linear mode, the amplitude of the output pulse is proportional to the amplitude of this input signal [1]. However, the performance of the SRO may be degraded by the noise perturbations, which may even give rise to non-negligible output pulses in the absence of an intended input signal. Thus, a reliable prediction of the signal-to-noise ratio $(\mathrm{S} / \mathrm{N})$ at the design stage is essential.

Several previous works [4]-[5] have presented a calculation of the signal-to-noise ratio of SROs in linear mode. However, most of them consider a simplified second-order system, usually described in terms of a cubic nonlinearity and a resonator. On the other hand, they do not accurately account for the cyclostationary properties of the circuit response to the noise sources, resulting from the action of the quench signal.
These properties enable an intuitive analysis of the cyclostationary process, which facilitates the calculation of both the output noise power spectral density (PSD) and the output noise power. The analysis presented here will make use of the numerical black-box model proposed in [6], which enables the determination of the SRO response to any arbitrarily modulated input signal. It is based on the calculation of an envelope-domain time-variant transfer function [7], relating the output and input of the SRO, and extracted from circuit-level envelope-transient simulations [8]-[9]. Here the black-box modelling technique will be used of obtain a linear time variant (LTV) transfer function with respect to each noise source. Then, a stochastic analysis will be carried out, taking into account the cyclostationary nature of the autocorrelation function of the output signal. This will enable an accurate prediction of the noise power spectral density and a straightforward determination of the signal-to-noise ratio.

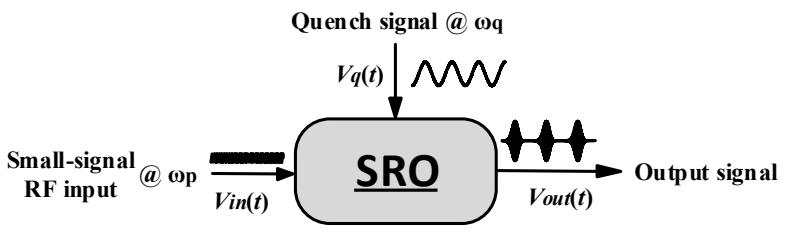

Fig. 1. Schematic representation of the SRO operation.

\section{NOISE ANALYSIS}

\section{A. LTV transfer functions with respect to the noise sources}

The envelope-domain analysis of the SRO is performed considering the oscillator free-running frequency $\omega_{p}$ as the carrier frequency. Then, the SRO output due to noise is expressed as:

$$
n_{\text {out }}(t)=\left|N_{\text {out }}(t)\right| \cos \left(\omega_{p} t+\varphi_{\text {out }}(t)\right)=\operatorname{Re}\left[N_{\text {out }}(t) e^{j \omega_{p} t}\right]
$$

In turn, the $M$ independent noise sources are expressed as $N_{m}(t) e^{j \omega_{p} t}$, where $m=1$ to $M$. To calculate the LTV transfer function [7] with respect to the noise source $N^{m}(t)$, this source is replaced with a deterministic small-signal source, having the general representation $G e^{j\left(\omega_{p}+\Omega\right) t}$, where the constant envelope $G$ may correspond to either a voltage or current. The LTV transfer function associated to this source is given by [7]:

$$
H^{m}\left(t, \Omega+\omega_{p}\right)=V_{\text {out }}\left(t, \Omega+\omega_{p}\right) / G
$$

where $V_{\text {out }}$ is the envelope of the output voltage. The above function is calculated sweeping $\Omega$ in $G e^{j\left(\omega_{p}+\Omega\right) t}$ and performing a circuit-level envelope transient analysis at each $\Omega$ step. 
Because the quench signal is periodic, $H^{m}\left(t, \Omega+\omega_{p}\right)$ is periodic too, with the same period $T_{q}$. To illustrate the meaning of $H^{m}\left(t, \Omega+\omega_{p}\right)$, the case of a single arbitrary noise source $N^{m}(t)$ is initially considered. The output envelope $N_{\text {out }}(t)$ due this source is calculated discretising the time variable in $H^{m}\left(t, \omega_{p}+\Omega\right)$ and performing an IFT integral at each time $t_{n}$ :

$$
N_{\text {out }}\left(t_{n}\right)=\frac{1}{2 \pi} \int_{\omega_{\min }-\omega_{p}}^{\omega_{\max }-\omega_{p}} H^{m}\left(t_{n}, \Omega+\omega_{p}\right) N^{m}(\Omega) e^{j \Omega t_{n}} d \Omega
$$

When the SRO operates in linear mode, only the device white noise sources will have a relevant effect.

\section{B. Stochastic analysis of the SRO output}

Let a noise source with the envelope $N(t)$ be considered, where the superscript $m$ has been dropped for notation clarity. For simplicity, the integration frequency interval about $\omega_{p}$ will be assumed to be symmetrical, so expression (3) is rewritten as:

$$
N_{\text {out }}(t)=\frac{1}{2 \pi} \int_{-B / 2}^{B / 2} H\left(t, \Omega+\omega_{p}\right) N(\Omega) e^{j \Omega t} d \Omega
$$

where $N(\Omega)=N_{r}(\Omega)+j N_{i}(\Omega)$ and $B$ is the input noise bandwidth. To obtain the power spectral density (PSD) of the process $N_{\text {out }}(t)$ one must first calculate the correlation function $R(t, \tau)$, with a double time dependence. In the case of a white-noise source $N(t)$, this is obtained as:

$$
\begin{aligned}
& R(t, \tau)=E\left[N_{\text {out }}(t+\tau) N_{\text {out }}(t)^{*}\right]= \\
& =\Gamma \int_{-B / 2}^{B / 2} \int_{-B / 2}^{B / 2} R_{H}\left(t, \tau ; \Omega_{1}, \Omega_{2}\right) \delta\left(\Omega_{1}-\Omega_{2}\right) e^{\left.j\left(\Omega_{1}-\Omega_{2}\right) t+\Omega_{1} \tau\right)} d \Omega_{1} d \Omega_{2}, \\
& R_{H}\left(t, \tau ; \Omega_{1}, \Omega_{2}\right) \equiv H\left(t+\tau, \Omega_{1}+\omega_{p}\right) H\left(t, \Omega_{2}+\omega_{p}\right)^{*} /(2 \pi)^{2}
\end{aligned}
$$

where $E\left[N\left(\Omega_{1}\right) N\left(\Omega_{2}\right)^{*}\right]=\Gamma \delta\left(\Omega_{1}-\Omega_{2}\right)$ and $\Gamma$ is the constant PSD. Operating the delta function, the above equation simplifies as:

$$
R(t, \tau)=\left(\frac{1}{2 \pi}\right)^{2} \Gamma \int_{-B / 2}^{B / 2} H\left(t+\tau, \Omega+\omega_{p}\right) H\left(t, \Omega+\omega_{p}\right)^{*} e^{j \Omega \tau} d \Omega
$$

Due to the time periodicity of $H\left(t, \Omega+\omega_{p}\right)$ for each $\Omega$, this function can be expanded in a Fourier series:

$$
H\left(t, \Omega+\omega_{p}\right)=\sum_{k} H_{k}(\Omega) e^{j k \omega_{q} t}, \quad \omega_{q}=2 \pi / T_{q}
$$

Replacing (7) into (6) one obtains:

$$
\begin{aligned}
& R(t, \tau)=E\left[N_{\text {out }}(t+\tau) N_{\text {out }}(t)^{*}\right]= \\
& =\frac{\Gamma}{(2 \pi)^{2}} \sum_{k, l}^{B / 2} \int_{-B / 2}^{B / 2} H_{k}(\Omega) H_{l}(\Omega)^{*} e^{j(k-l) \omega_{q} t} e^{j\left(k \omega_{q}+\Omega\right) \tau} d \Omega= \\
& =\sum_{k, l} U_{k, l}(\tau) e^{j(k-l) \omega_{q} t}=\sum_{s} R_{s}(\tau) e^{j s \omega_{q} t}, R_{s}(\tau) \equiv \sum_{k-l=s} U_{k, l}(\tau)
\end{aligned}
$$

where the components $U_{k, l}(\tau)$ are given by:

$$
U_{k, l}(\tau)=\frac{\Gamma}{(2 \pi)^{2}} \int_{-B / 2}^{B / 2} H_{k}(\Omega) H_{l}(\Omega)^{*} e^{j\left(k \omega_{q}+\Omega\right) \tau} d \Omega
$$

From (8), the autocorrelation function $R(t, \tau)$ satisfies:

$$
R(t, \tau)=R\left(t+T_{q}, \tau\right)
$$

On the other hand, and taking into account the periodicity of $H\left(t, \Omega+\omega_{p}\right)$, it is easily seen from (4) that the time average $E\left[N_{\text {out }}(t)\right]$ satisfies:

$$
E\left[N_{\text {out }}(t)\right]=E\left[N_{\text {out }}\left(t+T_{q}\right)\right]
$$

The fulfilment of (10) and (11) indicates that $N_{\text {out }}(t)$ is a cyclostationary stochastic process. Then, the PSD of this process can be calculated using the Fourier series expansion (8) of the correlation function as [10]-[11]:

$$
S(\Omega)=\left\langle\left|N_{\text {out }}(\Omega)\right|^{2}\right\rangle=\int_{-\infty}^{\infty} R_{0}(\tau) e^{-j \Omega \tau} d \tau
$$

This is because, as demonstrated in [10]-[11], only the term with $s=0$ of summation (8) contributes to the cyclostationary process PSD. Now, comparing (8) and (9), the term $R_{0}(\tau)$, which provides the output $\mathrm{PSD}$, is given by:

$$
\begin{aligned}
R_{0}(\tau) & =\sum_{k=-N}^{N} U_{k,-k}(\tau)=\frac{\Gamma}{(2 \pi)^{2}} \sum_{k=-P}^{P} e^{j k \omega_{q} \tau} g_{k}(\tau), \\
g_{k}(\tau) & \equiv \int_{-\infty}^{\infty}\left|H_{k}(\Omega)\right|^{2} e^{j \Omega \tau} d \Omega
\end{aligned}
$$

where it has been assumed that $H_{k}(\Omega) \approx 0$ for $|\Omega|>B / 2$. Finally, introducing (13) in (12), the PSD of the output noise is:

$$
\begin{aligned}
& S_{n}(\Omega)=\frac{\Gamma}{(2 \pi)^{2}} \sum_{k=-P}^{P} \int_{-\infty}^{\infty} g_{k}(\tau) e^{-j\left(\Omega-k \omega_{q}\right) \tau} d \tau= \\
& =\frac{\Gamma}{(2 \pi)^{2}} \sum_{k=-P}^{P}\left|H_{k}\left(\Omega-k \omega_{q}\right)\right|^{2}
\end{aligned}
$$

Expression (14) provides an intuitive approach to the output noise PSD $S_{n}(\Omega)$. Indeed, this equation shows that $S_{n}(\Omega)$ is shaped by the Fourier components $H_{k}(\Omega)$ of the LTV transfer function $H\left(t, \Omega+\omega_{p}\right)$. Note that the contribution of each component $H_{k}(\Omega)$ is shifted to the $k$-th harmonic of the quench frequency $\omega_{q}$.

In the case of $M$ white-noise sources, with the spectral densities $\Gamma_{m}$, where $m=1 \ldots M$, the output noise process in (4) would be given by the sum of all integral contributions corresponding to each input noise source. The analysis should be carried out in the same way as (4)-(14), producing a total output-noise spectral density of the form $S_{n}(\Omega)=\sum_{m, n} S_{n}^{m, n}(\Omega)$, with $S_{n}^{m, n}(\Omega)$ being the terms arising from the correlation between the $m$-th and $n$-th noise sources. 


\section{Signal to noise ratio}

To evaluate the $\mathrm{S} / \mathrm{N}$ ratio one should first obtain the noise power about the carrier frequency $\omega_{p}$. The case of a single white-noise source with the spectral density $\Gamma$ will be initially considered. An expression for the noise power can be derived using the result (14) for the output noise PSD as:

$$
\begin{aligned}
& P_{n}=\frac{\left\langle n_{\text {out }}(t)^{2}\right\rangle}{R}=\frac{\left\langle\left(\operatorname{Re}\left(N_{\text {out }}(t) e^{j \omega_{p} t}\right)\right)^{2}\right\rangle}{R}=\frac{\left\langle\left|N_{\text {out }}(t)\right|^{2}\right\rangle}{2 R}= \\
& =\frac{1}{2 R} \int_{-\infty}^{\infty} S_{n}(\Omega) d \Omega=\frac{1}{2 R} \frac{\Gamma}{(2 \pi)^{2}} \sum_{k} \int_{-\infty}^{\infty}\left|H_{k}\left(\Omega-k \omega_{q}\right)\right|^{2} d \Omega= \\
& =\frac{1}{2 R} \frac{\Gamma}{(2 \pi)^{2}} \int_{-\infty}^{\infty} \sigma_{H}^{2}(\Omega) d \Omega
\end{aligned}
$$

where expression (1) has been applied and $\sigma_{H}^{2}(\Omega)=\left\langle\left|H\left(t, \Omega+\omega_{p}\right)\right|^{2}\right\rangle$ is the time average of the square value of the LTV transfer function. Note that the mean square value of the envelope carrier contributes with the factor $\left\langle\sin ^{2} \omega_{p} t\right\rangle=\left\langle\cos ^{2} \omega_{p} t\right\rangle=1 / 2$ in (15). On the other hand, the signal power is obtained considering a single RF input tone, which will fulfill $V_{i n}(\Omega)=A \delta\left(\Omega-\Omega_{0}\right)$, where $\Omega_{0}$ represents a frequency offset from the carrier at $\omega_{p}$. Using the black-box model in [6], the signal power is:

$$
\begin{aligned}
& P=\frac{\left\langle\left|V_{\text {out }}(t)\right|^{2}\right\rangle}{2 R}=\frac{1}{2 R}\left\langle\left|\int_{-B / 2}^{B / 2} \frac{H^{i}\left(t, \Omega+\omega_{p}\right)}{2 \pi} V_{\text {in }}(\Omega) e^{j \Omega t} d \Omega\right|^{2}\right\rangle= \\
& =\frac{1}{2 R}\left(\frac{A}{2 \pi}\right)^{2}\left\langle\left|H^{i}\left(t, \Omega_{0}+\omega_{p}\right)\right|^{2}\right\rangle=\frac{1}{2 R}\left(\frac{A}{2 \pi}\right)^{2} \sigma_{H^{i}}^{2}\left(\Omega_{0}\right)
\end{aligned}
$$

where $H^{i}\left(t, \Omega+\omega_{p}\right)$ is the LTV transfer function with respect to the input signal. Finally, the signal-to-noise $(\mathrm{S} / \mathrm{N})$ ratio is calculated using (15) and (16) as:

$$
S / N=P / P_{n}=\frac{A^{2}}{\Gamma} \frac{\sigma_{H^{i}}^{2}\left(\Omega_{0}\right)}{\int_{-\infty}^{\infty} \sigma_{H}^{2}(\Omega) d \Omega}
$$

\section{APPLICATION TO A FET-BASED SRO}

The schematic and photograph of the FET-based SRO are shown in Fig. 2. This SRO operates at the oscillation frequency $f_{p}=2.7 \mathrm{GHz}$, with the quench signal frequency $f_{q}=8 \mathrm{MHz}$. The main white-noise contributions are due to the input $50 \mathrm{Ohm}$ resistor, modeled with a voltage source $N_{1}$, and the transistor noise, modelled with an equivalent drain-to-source current source $N_{2}$. The PSD of the input noise source and the transistor drain source are $\Gamma_{1}=8.10^{-19} \mathrm{~V}^{2} / \mathrm{Hz}$ and $\Gamma_{2}=4.10^{-20} \mathrm{~A}^{2} / \mathrm{Hz}$, respectively. Two envelope-domain LTV transfer functions $H^{m}\left(t, \Omega+\omega_{p}\right)$, where $m=1,2$, have been calculated with respect to these two noise sources. For illustration, Fig. 3(a) shows the transfer function with respect to the drain noise source (which is the dominant contribution), evaluated at $\Omega /(2 \pi)=-42.6 \mathrm{MHz}$. As can be seen, it is periodic at the quench-signal period and has a pulsed shape. The Fourier components $H_{k}(\Omega)$ of this signal for $k=0,1,2$ are shown in Fig. 3(b). The PSD of the output noise due to $N_{2}$ has been calculated using expression (14) with $P=32$ components. with the result of Fig. 4.

An input signal of power $P_{i n}=-60 \mathrm{dBm}$ and frequency offset $\Omega_{0} \approx 0$ will be considered. To obtain the $\mathrm{S} / \mathrm{N}$ ratio, the mean square values $\sigma_{H^{1,2}}^{2}(\Omega)$, corresponding to the noise sources, and $\sigma_{H^{1}}^{2}(0)=\left\langle\left|H^{1}\left(t, \omega_{p}\right)\right|^{2}\right\rangle$, corresponding to the RF input signal are calculated. The resulting values of $P_{n}, P$ and S/N using (15)-(17) are shown in Table I. In the following, these results will be compared with both circuit-level envelopetransient simulations and measurements of the noise response.

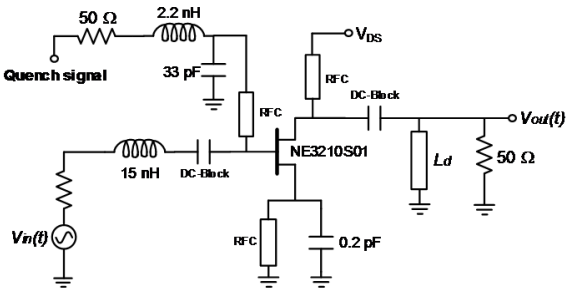

(a)

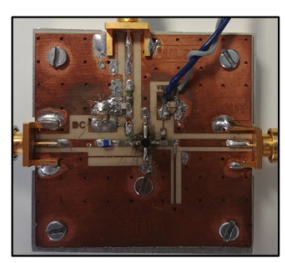

(b)
Fig. 2. Schematic and photograph of the FET-based SRO. The oscillation and quench frequency values are $f_{p}=2.7 \mathrm{GHz}$ and $f_{q}=8 \mathrm{MHz}$, respectively.
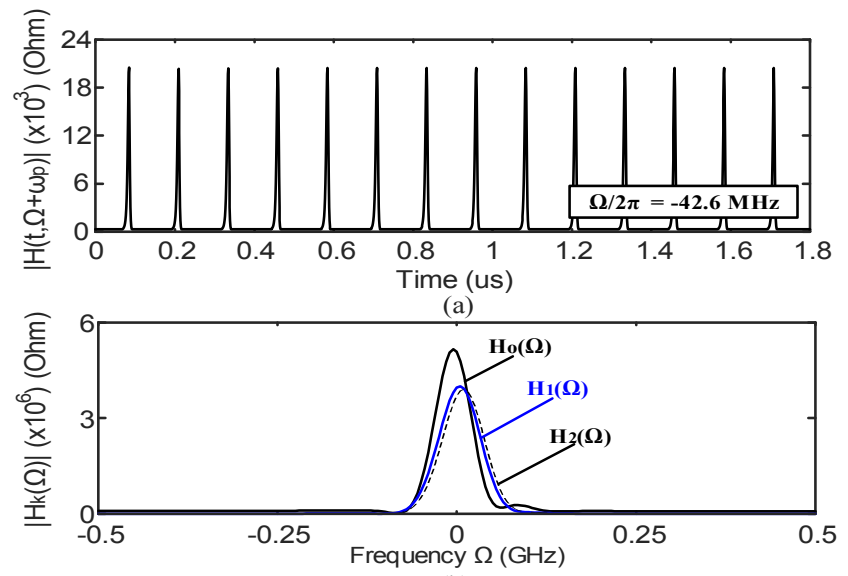

(b)

Fig. 3. Calculation of the output noise PSD. (a) Magnitude of the LTV transfer function $H^{2}\left(t, \Omega+\omega_{p}\right)$ evaluated at $\Omega /(2 \pi)=-42.6 \mathrm{MHz}$. This function is calculated with respect to the current noise source $N_{2}$. (b) Fourier coefficients $H_{k}(\Omega)$ of $H^{2}\left(t, \Omega+\omega_{p}\right)$, for $k=0,1,2$.

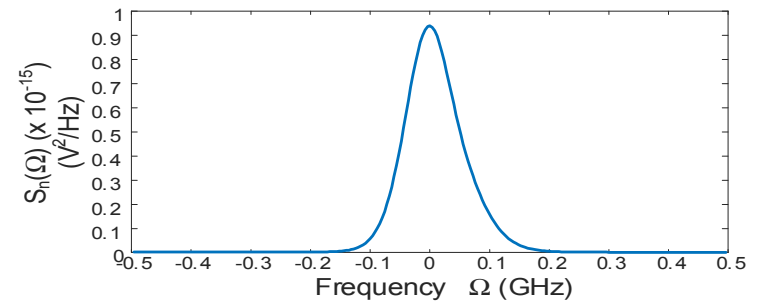

Fig. 4. PSD of the output noise due to a white-noise current source $N_{2}$. The PSD of the source is $\Gamma_{2}=4.10^{-20} \mathrm{~A}^{2} / \mathrm{Hz}$. 


\section{A. Numerical simulations}

From (15)-(16), the $\mathrm{S} / \mathrm{N}$ ratio can be expressed in terms of the mean square values of the envelope signals $V_{\text {out }}(t)$ and $N_{\text {out }}(t)$ as $S / N=\left\langle\left|V_{\text {out }}(t)\right|^{2}\right\rangle /\left\langle\left|N_{\text {out }}(t)\right|^{2}\right\rangle$. The mean square value $\left\langle\left|N_{\text {out }}(t)\right|^{2}\right\rangle$ is obtained through a costly circuit-level envelope-transient simulation, performed during a time interval of $12 \mu \mathrm{s}$. The output noise signal $N_{\text {out }}(t)$ is shown in Fig. 5(a). It presents oscillation pulses of small amplitude, due to the presence of noise perturbations in the SRO sensitivity interval. The resulting $\mathrm{S} / \mathrm{N}$ value is compared in Table I with the one provided by the stochastic analysis (15)-(16). There is a very good agreement between both analyses, with less than $1 \mathrm{~dB}$ of difference between the two calculations.

\section{B. Measurement results}

The experimental characterization of the SRO is initially performed without the RF deterministic input signal. The resulting output signal, due to noise perturbations only, is processed to extract the envelope of the output pulses, with the result of Fig. 5(b). The amplitude levels of the envelope pulses in $N_{\text {out }}(t)$ are very similar to those obtained in the simulation of Fig. 5(a). In the measurement, the $\mathrm{S} / \mathrm{N}$ value is directly calculated from the SRO output with and without input signal. The $\mathrm{S} / \mathrm{N}$ values obtained in measurement and simulation are compared in Table I.

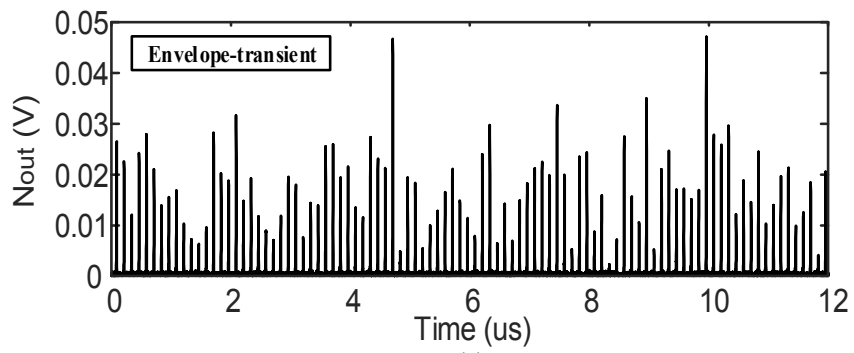

(a)

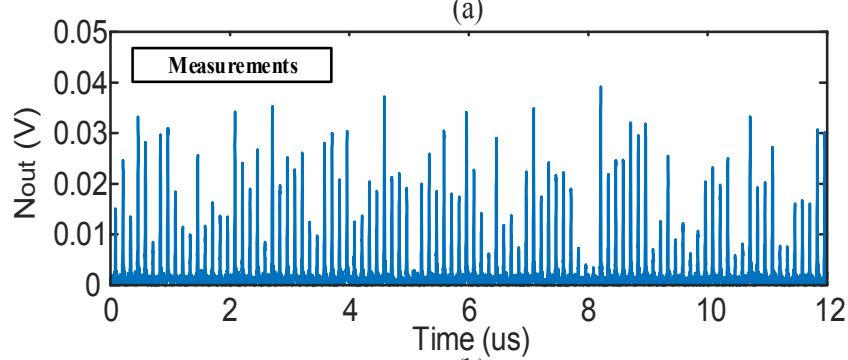

(b)

Fig. 5. Noise power calculation. Envelope of the output signal in the presence of noise perturbations only. (a) Envelope-transient simulation. (b) Measurements.

Table I. Signal to noise ratio calculation.

\begin{tabular}{|l|c|c|c|c|}
\hline & $\mathrm{P}(\mu \mathrm{W})$ & $\mathrm{P}_{\mathrm{n}}(\mu \mathrm{W})$ & $\mathrm{P}+\mathrm{P}_{\mathrm{n}}(\mu \mathrm{W})$ & $\mathrm{S} / \mathrm{N}(\mathrm{dB})$ \\
\hline Eq.(15)- Eq. (16) & 5.789 & 0.1071 & 5.896 & 17.32 \\
\hline Simulation & 5.786 & 0.1173 & 5.903 & 16.93 \\
\hline Measurements & - & 0.3241 & 13.23 & 16.01 \\
\hline
\end{tabular}

\section{CONCLUSION}

A stochastic analysis of the output signal of SROs in the presence of noise sources has been presented. It is applicable to SROs of arbitrary topology, since it is based on a black-box model of the SRO, in terms of a linear time variant (LTV) transfer function with respect to each noise source. The stochastic analysis takes into account the cyclostationary nature of the autocorrelation function of the output signal, enabling a straightforward determination of the output noise power spectral density and the signal-to-noise ratio. The results of the stochastic analysis have been successfully compared with circuit-level envelope-transient simulations, in the presence of noise sources, and with measurements.

\section{ACKNOWLEDGMENT}

This work was supported by the Spanish Ministry of Science, Innovation and Universities and the European Regional Development Fund (ERDF/FEDER) under the research project TEC2017-88242-C3-1-R.

\section{REFERENCES}

[1] F. X. Moncunill-Geniz, P. Pala-Schonwalder and O. Mas-Casals, "A generic approach to the theory of superregenerative reception," in IEEE Trans. Circuits Syst. I, Reg. Papers, vol. 52, no. 1, pp. 54-70, Jan. 2005.

[2] H. Ghaleb, P. V. Testa, S. Schumann, C. Carta and F. Ellinger, "A 160$\mathrm{GHz}$ Switched Injection-Locked Oscillator for Phase and Amplitude Regenerative Sampling," in IEEE Microw. Compon. Lett, vol. 27, no. 9, pp. 821-823, Sept. 2017.

[3] M. Vossiek and P. Gulden, "The Switched Injection-Locked Oscillator: A Novel Versatile Concept for Wireless Transponder and Localization Systems," IEEE Trans. Microw. Theory Techn., vol. 56, no. 4, pp. 859866, Apr., 2008.

[4] D. Lee and P. P. Mercier, "Noise Analysis of Phase-Demodulating Receivers Employing Super-Regenerative Amplification," in IEEE Transactions on Microwave Theory and Techniques, vol. 65, no. 9, pp. 3299-3311, Sept. 2017.

[5] P. E. Thoppay, C. Dehollain and M. J. Declercq, "Noise analysis in super-regenerative receiver systems," 2008 Ph.D. Research in Microelectronics and Electronics, Istanbul, 2008, pp. 189-192.

[6] S. Hernández and A. Suárez, "Envelope-Domain Analysis and Modeling of Super-Regenerative Oscillators," in IEEE Trans. Microw. Theory Techn., vol. 66, no. 8, pp. 3877-3893, Aug. 2018.

[7] L. A. Zadeh, "Frequency Analysis of Variable Networks," in Proceedings of the IRE, vol. 38, no. 3, pp. 291-299, March 1950.

[8] E. Ngoya and R. Larcheveque, "Envelop transient analysis: a new method for the transient and steady state analysis of microwave communication circuits and systems," IEEE MTT-S Int. Microwave Symp. Dig., San Francisco, CA, USA, 1996, vol.3, pp. 1365-1368.

[9] K. S. Kundert, "Introduction to RF simulation and its application," in IEEE Journal of Solid-State Circuits, vol. 34, no. 9, pp. 1298-1319, Sep 1999.

[10] J. Roychowdhury, D. Long and P. Feldmann, "Cyclostationary Noise Analysis of Large RF Circuits with Multitone Excitations", IEEE Journal of Solid-State Circuits, vol. 33, no. 3, March 1998

[11] T. Ström and S. Signell, "Analysis of periodically switched linear circuits," IEEE Trans. Circuits Syst., vol. CAS-24, pp. 531-541, Oct. 1977.S. Zhang, C. Zhu, J. K. O. Sin, and P. K. T. Mok, "A novel ultrathin elevated channel low-temperature poly-Si TFT," IEEE Electron Device Lett., vol. 20, pp. 569-571, Nov. 1999. 